human reproduction

\title{
A double-blind, non-inferiority RCT comparing corifollitropin alfa and recombinant FSH during the first seven days of ovarian stimulation using a GnRH antagonist protocol
}

\author{
P. Devroey', R. Boostanfar'2, N.P. Koper ${ }^{3}$, B.M.J.L. Mannaerts ${ }^{3,6}$, \\ P.C. IJzerman-Boon ${ }^{4}$, and B.C.J.M. Fauser ${ }^{5}$ on behalf of the ENGAGE \\ Investigators ${ }^{\dagger}$
}

'Center for Reproductive Medicine, UZ Brussel, Brussels, Belgium ${ }^{2}$ Huntington Reproductive Center, Westlake Village, CA, USA ${ }^{3}$ Global Clinical Research, Schering-Plough Research Institute, Molenstraat I I0, PO Box 20, Oss, The Netherlands ${ }^{4}$ Research Data and Quantitative Sciences, Schering-Plough Research Institute, Oss, The Netherlands ${ }^{5}$ Department of Reproductive Medicine and Gynecology, University Medical Center, Utrecht, The Netherlands

${ }^{6}$ Correspondence address. E-mail: b.mannaerts@spcorp.com

\begin{abstract}
BACKGROUND: Corifollitropin alfa, a fusion protein lacking LH activity, has a longer elimination half-life and extended time to peak levels than recombinant FSH ( $\mathrm{rFSH})$. A single injection of corifollitropin alfa may replace seven daily gonadotrophin injections during the first week of ovarian stimulation.
\end{abstract}

\begin{abstract}
METHODS: In this large, double-blind, randomized, non-inferiority trial the ongoing pregnancy rates were assessed after one injection of I $50 \mu \mathrm{g}$ corifollitropin alfa during the first week of stimulation and compared with daily injections of $200 \mathrm{IU}$ rFSH using a standard GnRH antagonist protocol.
\end{abstract}

RESULTS: The study population comprised 1506 treated patients with mean age of 31.5 years and body weight of $68.6 \mathrm{~kg}$. Ongoing pregnancy rates of $38.9 \%$ for the corifollitropin alfa group and $38.1 \%$ for $r F S H$ were achieved, with an estimated non-significant difference of $0.9 \%$ [95\% confidence interval $(\mathrm{Cl})$ : $-3.9 ; 5.7]$ in favor of corifollitropin alfa. Stratified analyses of pregnancy rates confirmed robustness of this primary outcome by showing similar results regardless of IVF or ICSI, or number of embryos transferred. A slightly higher follicular response with corifollitropin alfa resulted in a higher number of cumulus-oocyte-complexes compared with rFSH [estimated difference 1.2 (95\% Cl: 0.5; I.9)], whereas median duration of stimulation was equal ( 9 days) and incidence of (moderate/severe) ovarian hyperstimulation syndrome was the same (4.I and $2.7 \%$, respectively $P=0.15$ ).

CONCLUSION: Corifollitropin alfa is a novel and effective treatment option for potential normal responder patients undergoing ovarian stimulation with $\mathrm{GnRH}$ antagonist co-treatment for IVF resulting in a high ongoing pregnancy rate, equal to that achieved with daily $\mathrm{rFSH}$.

The trial was registered under ClinicalTrials.gov identifier NTC00696800.

Key words: corifollitropin alfa / sustained follicle stimulant / FSH / ovarian stimulation / IVF

† ENGAGE investigators: Belgium: Devroey, UZ Brusssel, Center for Reproductive Medicine, Brussels; Dhont, University Hospital Ghent, Department of Gynecology. Canada: Leader, The Ottawa Fertility Center, Ottawa, Ontario. Czech Republic: Mardesic, Sanatorium Pronatal, Prague; Mrázek, ISCARE IVF a.s., Prague. Denmark: Blaabjerg, Herlev Hospital, Fertility Clinic, Herlev. Finland: Tapanainen, Naistentautien klinikka, Oulun yliopistollinen sairaala (OYS), Oulu; Varila, Väestölitto, Tampereen klinikka, Tampere. France: Barrière, Hôpital de la mère et de l'enfant, Nantes; Hedon, Hôpital Arnaud de Villeneuve, Montpellier. The Netherlands: Fauser and Sterrenburg, University Medical Center, Department of Reproductive Medicine \& Gynecology, Utrecht. Norway: Kahn, Sykehuset Telemark HF, Skien; Von Düring, St. Olavs Hospital HF, Trondheim. Spain: Bajo Arenas, Ginefiv, Madrid; Barri, Institut Universitari Dexeus, Barcelona; Fernández-Sánchez, IVI Sevilla, Sevilla. Sweden: Bergh, Kvinnokliniken, Sahlgrenska Universitetssjukhuset, Göteborg; Hillensjö, Fertilitetscentrum, Carlanderska Sjukhuset, Göteborg. United Kingdom: Balen, Assisted Conception Unit, Leeds General Infirmary; Ledger, Assisted Conception Unit, Jessop Wing, The Halllamshire Hospital, Sheffield; Matthews, Bourn Hall Clinic, Cambridge. United States of America: Abuzeid, IVF Michigan, Rochester Hills (MI), Alper, Boston IVF, Waltham (MA); Boostanfar, Huntington Reproductive Center, Westlake Village (CA); Doody, Center for Assisted Reproduction, Bedford (TX); Frattarelli, Reproductive Medicine Associates of New Jersey, Morristown (NJ); Grunfeld, Reproductive Medicine Associates of New York, New York (NY); Karande, Karande and Associates SC, Hoffman Estates (IL); Kort, Reproductive Biology Associates, Atlanta (GA); Levy, Shady Grove Fertility Reproductive Science Center, Rockville (MD); Lifchez, Fertility Centers of Illinois, Chicago (IL); Pang, Reproductive Science Center of Boston, Lexington (MA); Schoolcraft, Colorado Center for Reproductive Medicine, Englewood (CO); Yeko, The Reproductive Medicine Group, Tampa (FL).

(C) The Author 2009. Published by Oxford University Press on behalf of the European Society of Human Reproduction and Embryology

This is an Open Access article distributed under the terms of the Creative Commons Attribution Non-Commercial License (http://creativecommons.org/licenses/by-nc/2.5/uk/) which permits unrestricted non-commercial use, distribution, and reproduction in any medium, provided the original work is properly cited. 


\section{Introduction}

Corifollitropin alfa is the first hybrid molecule with sustained follicle-stimulating activity. Corifollitropin alfa is a recombinant fusion protein composed of FSH and the carboxy terminal peptide (CTP) of the hCG $\beta$-subunit (Fares et al., 1992). Like recombinant FSH (rFSH), corifollitropin alfa interacts only with the $\mathrm{FSH}$-receptor and lacks $\mathrm{LH}$ activity (Lapolt et al., 1992; Fauser et al., 2009). However, corifollitropin alfa has an approximately 2 -fold longer elimination half-life $\left(t_{1 / 2}\right)$ and an almost 4-fold extended time-interval $\left(t_{\max }\right)$ to peak serum levels $\left(C_{\max }\right)$ (Duijkers et al., 2002). Due to this pharmacokinetic profile, corifollitropin alfa can function as a sustained follicle stimulant with a similar pharmacodynamic profile as $\mathrm{rFSH}$, but with the ability to initiate and sustain multiple follicular growth for an entire week. Consequently, a single s.c. injection of the recommended dose of corifollitropin alfa can replace the first seven injections of any daily FSH preparation in an ovarian stimulation treatment cycle prior to IVF.

The need for simplified treatment approaches which will lessen the treatment burden of IVF is self-evident. The IVF treatment process itself is increasingly recognized as contributing to the physical, psychological and emotional burden on infertility patients (Boivin and Takefman, 1996; Cousineau and Domar, 2007). Infertile patients experience high levels of distress and their level of anxiety and depression is equivalent to that experienced by women with cancer or heart disease (Domar et al., 1993). A number of IVF studies in which treatment costs were reimbursed have nevertheless reported drop-out rates well above $50 \%$ before completing their covered number of cycles (Land et al., 1997; Olivius et al., 2002; Schröder et al., 2004), largely due to the psychological impact of treatment (Olivius et al., 2004; Rajhkowa et al., 2006). Thus, the primary reason for treatment discontinuation is not based on physician recommendation, but is because patients are too distressed to continue (Hammarberg et al., 200 I).

In $\mathrm{GnRH}$ antagonist co-treatment stimulation protocols, the duration of stimulation is reduced compared with GnRH agonist protocols and less FSH is used to reach the same criteria for administering hCG (Tarlatzis et al., 2006). Interestingly, a prospective cohort study comparing a GnRH antagonist protocol with a conventional long $\mathrm{GnRH}$ agonist protocol demonstrated a significantly reduced drop-out rate in the antagonist group, indicating that the impact of the treatment strategy is an important factor determining the risk of drop-out (Verberg et al., 2008). Clearly, simple treatment regimens that lessen the burden of IVF improve the overall patient experience, and encourage lower drop-out rates (Olivennes 2003, Heijnen et al., 2004, Pennings and Ombelet, 2007). Last but not least, fewer injections to be given may improve drug compliance and/or prevent errors during drug administration.

Developing corifollitropin alfa in a short $\mathrm{GnRH}$ antagonist protocol may add to the further reduction of the treatment intensity experienced by patients undergoing ovarian stimulation for IVF. Following the dose-finding trial (The Corifollitropin alfa Dose-finding Study Group, 2008) and subsequent pharmacokinetic/pharmacodynamic modeling (De Greef et al., 2007) it was concluded that the recommended dose of corifollitropin alfa was $100 \mu \mathrm{g}$ for subjects with body weight of $\leq 60 \mathrm{~kg}$ and I $50 \mu \mathrm{g}$ for subjects with body weight of $>60 \mathrm{~kg}$. Based on simulations, these two dosages of corifollitropin alfa will provide the same exposure and the same degree of ovarian response in the recommended body weight groups. After a single injection of corifollitropin alfa on menstrual cycle day 2 or 3, treatment may be continued with a daily dose of rFSH from stimulation day 8 onwards if needed. Patients who reach the criteria of triggering final oocyte maturation prior to day 8 of stimulation do not require any daily FSH to be administered.

First and foremost the question that needs to be addressed is whether the new corifollitropin alfa regimen results in the same success rates as a daily FSH regimen with $\mathrm{GnRH}$ antagonist co-treatment. To this end, the aim of the ENGAGE trial was to investigate whether the ongoing pregnancy rates of the new corifollitropin alfa regimen were comparable to a daily $\mathrm{rFSH}$ regimen in patients undergoing ovarian stimulation prior to IVF. The sample size required to sufficiently power this trial for ongoing pregnancy rate as a primary end-point renders this the largest double-blind randomized trial in the field of assisted reproductive technology (ART) to date.

\section{Materials and Methods}

The ENGAGE trial was a multi-center, randomized, double-blind doubledummy, non-inferiority clinical trial involving 14 centers in North America ( 13 centers in USA and one in Canada) and 20 centers in Europe (three in Spain and The UK; two in Belgium, Czech Republic, Finland, France, Norway, and Sweden; one in Denmark and The Netherlands) and conducted between June 2006 and January 2008.

The study was approved by the Independent Medical Ethics Committee or Institutional Review Board for each center as well as by the responsible Health Authority and was conducted in accordance with the Declaration of Helsinki, International Conference on Harmonization guidelines for Good Clinical Practice, and local regulatory requirements. An Independent Data Safety Monitoring Board was appointed to monitor the safety of subjects participating in the trial, and written informed consent was provided by all patients.

\section{Study population}

Women aged 18-36 years with a body weight of more than $60 \mathrm{~kg}$ up to and including $90 \mathrm{~kg}$, a BMl of $18-32 \mathrm{~kg} / \mathrm{m}^{2}$, a menstrual cycle length of 24-35 days, access to ejaculatory sperm and an indication for controlled ovarian stimulation (COS) before IVF or ICSI were eligible to enroll in the study. Patients who had a (history of) an endocrine abnormality, an abnormal outcome of blood biochemistry or hematology, an abnormal cervical smear, a chronic disease, relevant ovarian-, tubal- or uterine-pathology that could interfere with the $\cos$ treatment (e.g. endometrioma $>0 \mathrm{~mm}$ or fibroids $\geq 5 \mathrm{~cm}$ ), embryo implantation or pregnancy were not to be included in the trial. Patients who had a history of ovarian hyperresponse (more than 30 follicles $\geq 11 \mathrm{~mm}$ ) or ovarian hyperstimulation syndrome (OHSS), polycystic ovary syndrome (PCOS) or a basal antral follicle count (AFC) of more than 20 on ultrasound $(<1 \mathrm{Imm}$, both ovaries combined) were excluded from participation. Other exclusion criteria included a previously low ovarian response to FSH or hMG treatment (i.e. cycle cancelled due to insufficient ovarian response or less than four oocytes obtained), an FSH or LH over I $2 \mathrm{IU} / \mathrm{L}$ in the early follicular phase, more than three consecutive unsuccessful IVF cycles since the last ongoing pregnancy, a history of recurrent miscarriage (three or more), or currently smoking more than five cigarettes per day.

\section{Study design}

The trial was designed as a randomized, double-blind, double-dummy, active-controlled, non-inferiority trial to compare the efficacy of a single injection of corifollitropin alfa during the first week of stimulation with 
7 daily injections of $\mathrm{rFSH}$ for inducing and sustaining multifollicular growth during COS. Randomization to one of the two treatment arms (I:I ratio) was done per center and stratified by age ( $<32$ and $\geq 32$ years) by central remote allocation using randomly permutated blocks with an undisclosed fixed block size of four.

\section{Stimulation regimen and ART procedures}

All patients were to start their treatment cycle on menstrual cycle day 2 or 3 as depicted in Fig. I. Per protocol all injections were to be administered in the morning. Patients started stimulation with a single s.c. injection of $150 \mu \mathrm{g}(0.5 \mathrm{~mL})$ corifollitropin alfa (NV Organon, The Netherlands) or matching placebo. Injections could be done by the patient herself, her partner or the medical staff. To conceal treatment allocation all patients also started daily s.c. injection of $200 \mathrm{IU}$ rFSH (follitropin beta, Puregon ${ }^{\circledR} /$ Follistim $^{\circledR}$ AQ Cartridge, NV Organon, The Netherlands) or matching placebo on the same day (Stimulation Day I) using the Puregon ${ }^{\circledR} /$ Follistim Pen ${ }^{\circledR}$. Daily active or placebo ('dummy') rFSH injections were continued through the first 7 days of stimulation. The chosen reference dose of $200 \mathrm{IU}$ rFSH daily was considered the optimal choice for the included patient population weighing over $60 \mathrm{~kg}$ in a global trial combining European and North American sites. This dose was fixed for the first 5 days of stimulation. This is in line with the suggestion by Arce et al. (2005) who recommended a fixed starting dose for at least 5-7 days in all efficacy trials as only after such period the impact of the administered FSH dose can be adequately evaluated. Moreover, this is considered appropriate because daily FSH only reaches steady state after 3-5 days of dosing (Mannaerts et al., 1993). A reduction of the rFSH dose was allowed from stimulation day 6 onward in case of too high an ovarian response, at the discretion of the investigator. When no follicle $\geq I \mathrm{I} \mathrm{mm}$ was visible on ultrasound scan (USS) before injection on stimulation day 8 the cycle was to be cancelled due to insufficient ovarian response. From stimulation day 8 onwards, treatment in both groups was continued with a daily s.c. dose of (active) rFSH up to and including the day of hCG administration. The maximum rFSH dose to continue treatment after the first 7 days was $200 \mathrm{IU}$ but the dose could be reduced when desired. For normal responders, the recommended daily dose of $\mathrm{rFSH}$ was $150 \mathrm{IU}$. Whenever deemed required by the investigator, rFSH administration could be withheld for a maximum of 3 days (coasting) up to and including the day of hCG administration. In case there was a too high ovarian response as per the investigator's opinion, the cycle could be cancelled at any time. However, in case of a risk for OHSS, defined as more than 30 follicles of $\geq 11 \mathrm{~mm}$ on USS, hCG was always to be withheld and the treatment cycle was to be cancelled per protocol. The maximum total duration of stimulation was 19 days.

To prevent premature LH surges the $\mathrm{GnRH}$ antagonist ganirelix $(0.25 \mathrm{mg}$, Orgalutran ${ }^{\circledR}$ /ganirelix acetate injection, NV Organon, The Netherlands) was administered once daily s.c. starting on stimulation day 5 up to and including the day of hCG. Urinary hCG (10 $000 \mathrm{IU})$ was administered to induce final oocyte maturation as soon as at least three follicles of $\geq 17 \mathrm{~mm}$ were observed by USS. Investigators were allowed to delay hCG administration for I day when preferred for practical reasons. In case there was a too high ovarian response in the opinion of the investigator, a lower dose (5000 IU hCG) could be used. About 34-36 h thereafter, oocyte retrieval followed by standard IVF or ICSI was to be performed. Embryo quality was evaluated for all available embryos on day 3 of culture by the local embryologist using a protocol-defined guideline based on the following parameters: number of blastomeres, degree of fragmentation, blastomere size uniformity and presence or absence of multinucleation. Embryos graded as grade I (6- 10 cells, no fragmentation and equal blastomere size) or grade 2 (allowing up to $20 \%$ fragmentation) were qualified as good quality embryos. The quality of embryos continued in culture after day 3 was reassessed on the day of transfer or freezing using grading criteria appropriate for the stage of embryo culture. At embryo transfer, 3 or 5 days after oocyte retrieval, one or two embryos were to be transferred. The decision on the day of transfer and number of embryos to be transferred was made by the investigator. To support implantation and early pregnancy, luteal phase support with progesterone (at least $600 \mathrm{mg} /$ day vaginally or at least $50 \mathrm{mg}$ /day i.m., to be prescribed locally) was started on the day of oocyte retrieval and continued for at least 6 weeks, or either up to menses or up to a negative pregnancy test performed at least 14 days after embryo transfer.

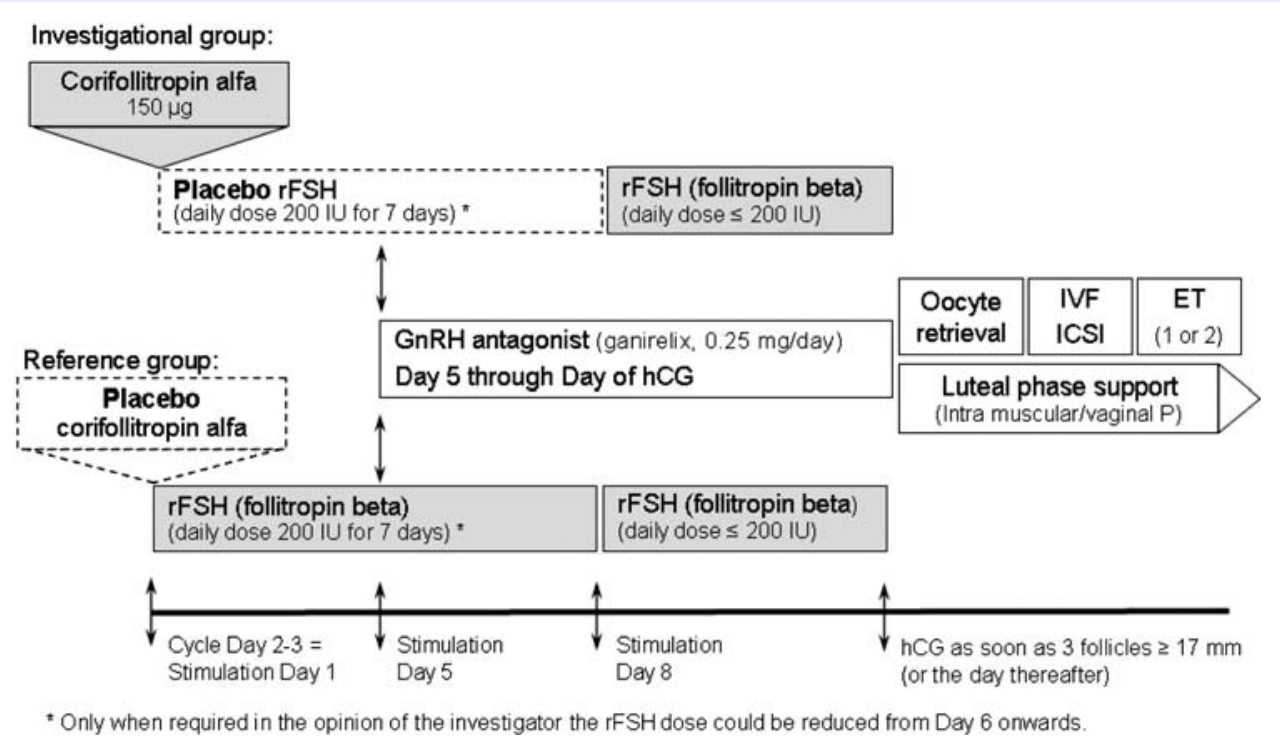

Figure I Graphical illustration of the treatment regimens applied in this trial. Upper panel depicts investigational group (corifollitropin alfa), lower panel depicts reference group (rFSH).

rFSH: recombinant FSH, P: progesterone. 


\section{Assessments}

Before the start of ovarian stimulation, pregnancy was excluded by means of an hCG test, a blood sample was obtained for hormone assessments, and USS was performed to measure and count visible follicles. Local tolerance parameters (pain, itching, swelling and redness) were assessed by the clinical staff $30 \mathrm{~min}$ after injection for both (placebo) corifollitropin alfa and (placebo) rFSH injection sites. Patients returned to the clinic for USS and blood sampling on stimulation days 5 and 8 , and then daily up to and including the day of hCG administration for USS only. Additional blood samples were collected on the day of embryo transfer and 2 weeks after embryo transfer. Patients who left the study prior to embryo transfer were sampled for hormones and antibody assessments at the day of discontinuation and 2 weeks thereafter.

Validated immunoassays were performed at a central laboratory (Schering-Plough, Oss, The Netherlands and Waltrop, Germany) to measure serum levels of $\mathrm{FSH}, \mathrm{LH}$, estradiol $\left(\mathrm{E}_{2}\right)$, progesterone, inhibin-B and antibodies against corifollitropin alfa (Devroey et al., 2004).

\section{End-points}

Ongoing pregnancy, defined as presence of at least one fetus with heart activity at least 10 weeks after embryo transfer as assessed by USS or Doppler, or confirmed by live birth, was the primary end-point for this trial. This can be considered as the best and closest estimate of the ultimate treatment success (delivery of a healthy baby) and excludes subjects with a miscarriage during the first 10 weeks of pregnancy (Arce et al., 2005). In addition, the number of retrieved oocytes was considered as co-primary end-point in this trial being more proximately related to the pharmacological effect of the two treatment regimens which are compared in this trial. Other clinical outcome parameters evaluated included dose of rFSH required, duration of stimulation, number and size of follicles, serum hormone levels, fertilization rate, number and quality of embryos obtained and pregnancy rates. The ultimate live birth rate, the health of the offspring, and outcomes achieved with spare, frozen embryos will be reported separately when follow-up has been completed.

Occurrence of (serious) adverse events, including moderate and severe OHSS as per World Health Organization criteria (WHO, 1973), outcome of local tolerance and immune response assessments were evaluated as safety end-points.

\section{Statistical analysis}

The sample size needed for this trial was largely determined by the chosen pre-defined non-inferiority margin (i.e. smaller margin requires larger sample size to maintain the same power) but also depended on the anticipated ongoing pregnancy rate (i.e. higher pregnancy rate requires larger sample size) to be expected in a combined trial comprising sites in Europe, USA and Canada. For this global trial a non-inferiority margin of $8 \%$ was considered appropriate. This implies that if the lower bound of the $95 \%$ confidence interval $(\mathrm{Cl})$ for the estimated difference in ongoing pregnancy rates between treatment groups (corifollitropin alfa minus $\mathrm{rFSH}$ ) was determined to be above $-8 \%$, corifollitropin alfa could be considered non-inferior to $\mathrm{rFSH}$. Although such a difference would be relevant for an individual subject seeking to become pregnant after IVF treatment it should be considered in the context of existing differences in routine pregnancy rates between centers, countries and regions (Gleicher et al., 2006, 2007). A sample size of at least 1380 subjects was calculated to be the minimum required to demonstrate non-inferiority with a power of $90 \%$, using a $-8 \%$ non-inferiority margin for the lower limit of the two-sided $95 \% \mathrm{Cl}$, and assuming an ongoing pregnancy rate of $30 \%$. Based on these data, a minimum of 700 subjects per group, in total at least 1400 subjects, were to be randomized which makes this the largest double-blind comparative randomized trial performed to date.

For analysis of the primary end-point, ongoing pregnancy, the treatment groups were formally compared with a generalized linear model for the ongoing pregnancy rate including covariates treatment group, age ( $<32$ years, $\geq 32$ years) and region (Europe, North America). The difference between the two treatment groups (corifollitropin alfa - rFSH) and its associated two-sided $95 \%$ likelihood-based $\mathrm{Cl}$ was estimated and a predefined non-inferiority margin of $8 \%$ was applied. Additional explorative analyses were performed to investigate robustness of the primary endpoint results. No adjustment for multiplicity to correct for repeated testing was performed.

The number of cumulus-oocyte-complexes retrieved was defined as co-primary end-point in this trial. As there is an optimal range of oocytes obtained in response to ovarian stimulation, below and above which the success rates of IVF are compromised (Van der Gaast et al., 2006) equivalence testing was deemed appropriate. Therefore, equivalence margins for the difference in the number of oocytes retrieved were predefined to be -3 and +5 oocytes. If the new corifollitropin alfa regimen resulted in three or more oocytes less than the reference treatment, such difference was considered as clinically relevant because three oocytes usually result in one good quality embryo for transfer or freezing. Anticipating obtaining an average of $12-13$ oocytes with the applied rFSH doses in the reference group, an excess of more than five oocytes would be undesirable as patients with more than 18 oocytes retrieved are described to be at increased risk of developing OHSS (Papanikolaou et al., 2006; Verwoerd et al., 2008). Hence, an upper margin of +5 oocytes is applied for the difference in the number of oocytes retrieved between the treatment groups. The treatment groups were formally compared using analysis of variance for the number of oocytes including covariates treatment group, age ( $<32$ years versus $\geq 32$ years) and center. The estimate of the difference between the two treatment groups and its associated two-sided $95 \% \mathrm{Cl}$ was given. In case the $95 \%$ $\mathrm{Cl}$ of the difference exceeded -3 or +5 oocytes corifollitropin alfa treatment was not considered equivalent to the rFSH treatment.

Efficacy analyses are based on the intention-to-treat (ITT) population entailing all patients randomized and treated, analyzing them according to their allocated treatment (i.e. 'as randomized'). Both ITT and per protocol (PP) analyses were performed (see Supplementary Data), but as the results were very similar, only the ITT results are presented here. The main efficacy analyses were performed 'per started cycle' (i.e. including all subjects who started treatment regardless whether they discontinued) as this provides the most conservative efficacy estimates as it also accounts for any unintended interference of premature cycle cancellations. For safety end-point analyses, patients are grouped 'as treated' which implied that three patients (one randomized to corifollitropin alfa and two patients to $\mathrm{rSH}$ ) treated inadvertently with the wrong (comparator) medication type are analyzed according to the treatment they actually received. Statistical significance was set at $P<0.05$.

\section{Results}

A total of 1696 patients signed informed consent for eligibility evaluation and participation in this trial (Fig. 2). Subsequently a total of 187 patients failed screening or dropped out due to personal reasons prior to treatment allocation. Eventually, 1509 patients were randomized to one of the two treatment groups of which 1506 patients actually started stimulation. The remaining three patients were discontinued prior to the start of treatment (one for personal reasons and two were found to violate entry criteria after randomization but before commencing treatment). 


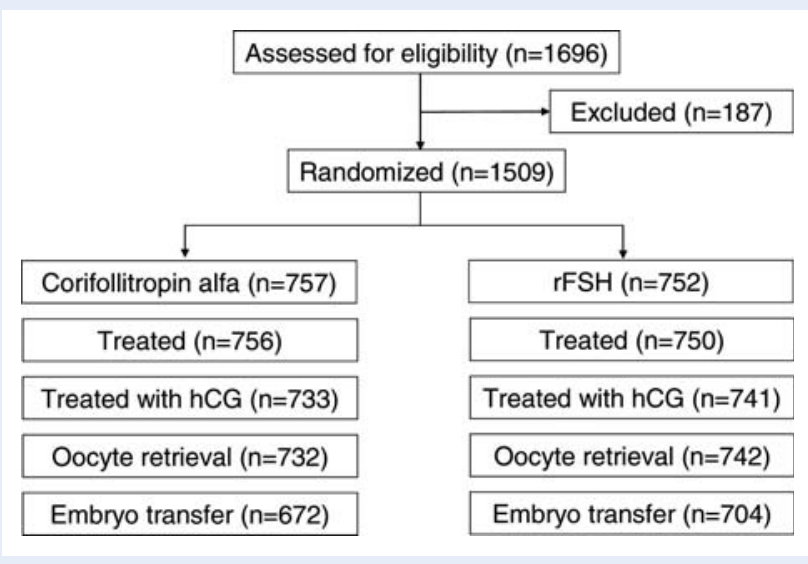

Figure 2 Flow chart showing the number of participants at each stage of the clinical trial.

Demographics of the ITT population as well as relevant fertility characteristics, USS findings and hormone profiles were comparable in the two groups (Table I). Mean age (SD) of the patients included in this trial was $31.5(3.3)$ years, body weight was $68.6(7.5) \mathrm{kg}$ and BMI was $24.8(2.7) \mathrm{kg} / \mathrm{m}^{2}$. The average duration of infertility was 3.3 (2.3) years and for $74 \%$ of the patients this was their first treatment cycle. On stimulation day I, no differences were observed between the treatment groups in the basal AFC or baseline serum hormone levels (Table I).

\section{Primary end-point: ongoing pregnancy rate}

Results of the primary end-point analyses are presented in Table II. High ongoing pregnancy rates of $38.9 \%$ for the corifollitropin alfa group and $38.1 \%$ for the $\mathrm{rFSH}$ group were obtained per started cycle. The estimated treatment difference, adjusted for age group $(<32$ years, $\geq 32$ years) and region (Europe, North America), was $+0.9 \%[95 \% \mathrm{Cl}:(-3.9 ; 5.7)]$ in favor of corifollitropin alfa.

\section{Co-primary end-point: number of cumulus- oocyte-complexes retrieved}

The number of cumulus-oocyte-complexes retrieved was defined as co-primary end-point in this trial. The mean (SD) number of oocytes retrieved in the corifollitropin alfa group was 13.7 (8.2) which was higher than the mean of 12.5 (6.7) obtained in the rFSH group (Table II). The estimated treatment difference was 1.2 oocytes in favor of corifollitropin alfa $(P=0.00 \mathrm{I})$, while the $95 \% \mathrm{Cl}$ was $(0.5$; 1.9). This indicates that the number of oocytes retrieved in the two treatment groups was at least equivalent based on the pre-defined equivalence range.

\section{Other clinical outcome parameters}

The median duration of stimulation was 9 days for both treatment groups, which implied patients treated with corifollitropin alfa needed on average 2 days of $r F S H$ to complete their treatment cycle (Table III). Per protocol, the dose of (placebo) rFSH could be reduced (not increased) from stimulation day 6 onwards. Dose decreases on stimulation days 6 or 7 were recorded slightly more

\begin{tabular}{|c|c|c|}
\hline & $\begin{array}{l}\text { Corifollitropin } \\
\text { alfa }(n=756)\end{array}$ & $\begin{array}{l}\text { rFSH } \\
(n=750)\end{array}$ \\
\hline \multicolumn{3}{|l|}{ Demographics } \\
\hline Age (years) & $31.5(3.3)$ & $31.5(3.2)$ \\
\hline Body weight (kg) & $68.8(7.6)$ & $68.4(7.3)$ \\
\hline $\mathrm{BMI}\left(\mathrm{kg} / \mathrm{m}^{2}\right)$ & $24.8(2.8)$ & $24.8(2.7)$ \\
\hline \multicolumn{3}{|l|}{ Race, $n(\%)$} \\
\hline Asian & $21(2.8)$ & $21(2.8)$ \\
\hline Black & $33(4.4)$ & $28(3.7)$ \\
\hline Caucasian & $643(85.1)$ & $650(86.7)$ \\
\hline Other & $59(7.8)$ & $5 \mathrm{I}(6.8)$ \\
\hline \multicolumn{3}{|l|}{ Fertility characteristics } \\
\hline Primary infertility, n (\%) & $403(53.3)$ & $393(52.4)$ \\
\hline Duration of infertility (years) & $3.3(2.4)$ & $3.2(2.2)$ \\
\hline \multicolumn{3}{|l|}{ Cause of infertility ${ }^{a)}, n(\%)$} \\
\hline Male factor & $388(5 \mid .3)$ & $347(46.3)$ \\
\hline Tubal factor & $198(26.2)$ & |9| (25.5) \\
\hline Endometriosis & $109(14.4)$ & $115(15.3)$ \\
\hline Other/unexplained & $246(32.5)$ & $262(34.9)$ \\
\hline First IVF cycle, $n(\%)$ & $569(75.3)$ & $552(73.6)$ \\
\hline \multicolumn{3}{|l|}{ Stimulation day I } \\
\hline Total ovarian volume $(\mathrm{mL})^{\mathrm{b})}$ & $13.2(8.1)$ & $13.2(7.1)$ \\
\hline Basal AFC $(<1 \mathrm{I} \mathrm{mm})$ & $12.3(4.6)$ & $12.4(4.4)$ \\
\hline FSH (IU/L) & $6.7(2.1)$ & $6.6(1.9)$ \\
\hline $\mathrm{LH}(\mathrm{IU} / \mathrm{L})$ & $4.8(2.0)$ & $4.7(1.8)$ \\
\hline $\mathrm{E}_{2}(\mathrm{pmol} / \mathrm{L})$ & I26.I (39.3) & I24.8 (37.4) \\
\hline Progesterone (nmol/L) & $1.8(\mathrm{I} .3)$ & I.8 (I.4) \\
\hline
\end{tabular}

AFC: antral follicle count, $E_{2}$ : estradiol, $r F S H$ : recombinant FSH. Numbers are mean (SD) unless otherwise indicated.

a) A patient can have multiple causes of infertility.

b) According to the formula for a polate ellipsoid: $0.523 \times$ longitudinal $\times$

antero-posterior $\times$ transverse diameters as measured per transvaginal ultrasound.

often in the corifollitropin alfa group $(85 / 750,11.3 \%)$ than in the rFSH group (62/74I, 8.4\%). Coasting by withholding rFSH for 2 or more days was applied in I.6\% [12/733, 95\% Cl: $(0.7 ; 2.6)]$ of patients in the corifollitropin alfa group and in $2.2 \%$ [16/74I, 95\% Cl: (I.0; 3.3)] of patients in the $\mathrm{rFSH}$ group. A fixed dose regimen from stimulation day 8 up to but not including the day of hCG was used in $72.1 \%$ $(413 / 573)$ of the corifollitropin alfa group and $81.0 \%$ (430/531) of the rFSH group. After a single injection of corifollitropin alfa, 249 out of 756 patients $(32.9 \%)$ reached the criteria for giving hCG before or on stimulation day 8: the ongoing pregnancy rate for this subgroup of patients was $44.0 \%$.

Eventually, $97.0 \%$ of the patients in the corifollitropin alfa group and $98.8 \%$ of the $\mathrm{rFSH}$ group received hCG to induce final oocyte maturation. The vast majority received 10000 IU hCG (76.9\% (58I/756) and $85.6 \%(642 / 750)$ of the patients in the corifollitropin alfa and rFSH group, respectively), while a dose of 5000 IU hCG was used 
Table II Primary end-points of the ENGAGE trial: ongoing pregnancy rate (assessed at least 10 weeks after embryo transfer) and the mean (SD) number of cumulus-oocyte-complexes retrieved (intent-to-treat population)

\begin{tabular}{|c|c|c|c|}
\hline & Corifollitropin alfa $(n=756)$ & $\mathrm{rFSH}(n=750)$ & Estimated difference $\left.^{a}\right)[95 \% \mathrm{Cl}], P$-value \\
\hline Ongoing pregnancies (n) & 294 & 286 & \\
\hline Per started cycle (\%) & $38.9 \%$ & $38.1 \%$ & $0.9[-3.9 ; 5.7], P=0.71$ \\
\hline Per embryo transfer (\%) & $43.8 \%$ & $40.6 \%$ & $3.1[-2.0 ; 8.2], P=0.24$ \\
\hline \multicolumn{4}{|l|}{ Cumulus-oocyte-complexes } \\
\hline Per started cycle & I3.7 (8.2) & $12.5(6.7)$ & $1.2[0.5 ; 1.9], P=0.001$ \\
\hline Per oocyte retrieval & |4.| (7.9) & $12.7(6.7)$ & $1.6[0.8 ; 2.3], P<0.001$ \\
\hline
\end{tabular}

$P$-value corresponds to the test whether the treatment difference equals zero.

a) Estimated treatment difference (corifollitropin alfa $-\mathrm{rFSH}$ ) adjusted for covariates.

Table III Clinical parameters from stimulation phase up to embryo transfer (intent-to-treat population)

\begin{tabular}{|c|c|c|}
\hline & Corifollitropin alfa $(n=756)$, median (range) & rFSH $(n=750)$, median (range) \\
\hline \multicolumn{3}{|l|}{ Stimulation characteristics ${ }^{a)}$} \\
\hline Total dose of rFSH (IU) & $400(0-2000)$ & $1800(400-2800)$ \\
\hline Total dose of rFSH from day 8 onwards (IU) & $400(0-2000)$ & $400(0-1400)$ \\
\hline Total duration of stimulation (days) ${ }^{\text {b) }}$ & $9(6-18)$ & $9(6-15)$ \\
\hline \multicolumn{3}{|l|}{ Follicles, day of $\mathrm{hCG}^{\mathrm{a})}$} \\
\hline$\geq 11 \mathrm{~mm}$ & $16.0(7.0)$ & $13.9(6.1)$ \\
\hline$\geq 15 \mathrm{~mm}$ & $9.6(4.8)$ & $8.7(4.0)$ \\
\hline$\geq 17 \mathrm{~mm}$ & $5.7(3.2)$ & $5.6(2.9)$ \\
\hline \multicolumn{3}{|l|}{ Serum parameters, day of $\mathrm{hCG}^{\mathrm{a})}$} \\
\hline FSH (IU/L) & $12.5(3.3)$ & $11.6(2.8)$ \\
\hline $\mathrm{LH}(\mathrm{IU} / \mathrm{L})$ & I.4 (।.8) & $1.9(1.6)$ \\
\hline $\mathrm{E}_{2}(\mathrm{pmol} / \mathrm{L})$ & $5508.8(3469.8)$ & $5165.3(2998.2)$ \\
\hline Inhibin-B (pg/mL) & $610.3(492.3)$ & $614.8(435.6)$ \\
\hline Progesterone (nmol/L) & $3.0(2.1)$ & $3.2(1.5)$ \\
\hline \multicolumn{3}{|l|}{ Clinical outcome per started cycle } \\
\hline Oocytes retrieved, ICSI only & I3.8 (7.6) & $12.1(6.3)$ \\
\hline Metaphase II oocytes (ICSI only), \% of total & $10.8(6.5), 78.9(18.9)$ & $9.2(5.1), 77.4(18.1)$ \\
\hline Fertilization rate (\%) ${ }^{\mathrm{c}, \mathrm{d})}$ & $66.0(23.4)$ & $67.6(22.9)$ \\
\hline Total number of embryos obtained (day 3$)^{c}$ & $8.3(5.6)$ & $7.4(4.8)$ \\
\hline Excellent (top) quality embryos (grade I) ${ }^{c}$ & $2.6(3.4)$ & $2.5(3.4)$ \\
\hline Good quality embryos (grade $I+2)^{c}$ & $4.6(4.3)$ & $4.4(3.9)$ \\
\hline Single embryo transfer (\%) $\left.{ }^{d}\right)$ & 25.7 & 27.0 \\
\hline Embryos transferred ${ }^{\mathrm{e}}$ & $1.7(0.4)$ & $1.7(0.4)$ \\
\hline Embryos cryopreserved ${ }^{f)}$ & $4.3(3.6)$ & $3.9(2.7)$ \\
\hline
\end{tabular}

Numbers are mean (SD) unless otherwise indicated.

a) Restricted to patients with hCG injection.

b) Number of days up to and including the day of hCG administration.

${ }^{c}$ Restricted to patients with IVF and/or ICSI.

d) Defined as 100 times the number of mature oocytes (with two pronuclei) obtained divided by the number of oocytes used for fertilization.

e) Restricted to patients with embryo transfer.

${ }^{\text {f) }}$ Restricted to patients with cryopreserved embryos.

for $19.8 \%$ (150/756) and $13.1 \%(98 / 750)$ of the respective groups. Only the most frequent reasons for discontinuation prior to hCG are mentioned. In total, 23 (3.0\%) of the patients in the corifollitropin alfa group and $7(0.9 \%)$ of the patients in the $\mathrm{rFSH}$ group discontinued prior to hCG. In the rFSH group, 2 patients discontinued after the oocyte retrieval procedure, but it appeared that they had not received hCG. Taking this into account, totals add up correctly to the number of subjects treated with hCG $(733=756-23,74 I=750-7-2)$. 
On the day of hCG, the mean (SD) number of follicles measuring $\geq I I \mathrm{~mm}$ on USS was 16.0 (7.0) for the corifollitropin alfa group and I3.9 (6.I) for the rFSH reference group (Table III). When comparing serum hormones on the day of hCG administration comparable levels were observed in both treatment groups. Oocyte retrieval was performed in $96.8 \%$ of the patients in the corifollitropin alfa group and $98.9 \%$ in the rFSH group. A higher mean number of cumulus-oocytecomplexes was retrieved in the corifollitropin alfa group (Table II). Oocyte maturity was assessed for ICSI patients and also showed a higher number and percentage of mature oocytes (Table III) in the corifollitropin alfa group (mean 10.8, 78.9\%) compared with the rFSH group (mean 9.2, 77.4\%). A comparable fertilization rate was observed between the groups resulting in a mean (SD) of 8.3 (5.6) embryos obtained on day 3 of culture in the corifollitropin alfa group and 7.4 (4.8) embryos in the rFSH group for patients with IVF and/or ICSI. Embryo quality, as assessed by the local embryologist, was similar in both groups.

Ultimately, 672 patients (88.9\%) in the corifollitropin alfa group and 704 patients $(93.9 \%)$ in the $\mathrm{rFSH}$ group had embryo transfer in this trial. Between hCG administration and embryo transfer 39 out of 756 patients $(5.2 \%)$ in the corifollitropin alfa group and 34 out of 750 (4.5\%) patients in the rFSH group were discontinued as a result of too few or too low quality oocytes, lack of fertilization or poor embryo development. During this period $6 / 756$ patients $(0.8 \%)$ in the corifollitropin alfa group and none in the rFSH group were discontinued due to a too high ovarian response or risk of OHSS. Although no embryo transfer was performed, fertilized oocytes or embryos were cryopreserved for all these six patients. When embryo transfer was performed, single embryo transfer was carried out in slightly more than a quarter of all transfers (Table III). On average, I.7 (0.4) embryos were transferred in both treatment groups. For 51.7 and $53.2 \%$ of the patients in the corifollitropin alfa and rFSH groups, a mean (SD) of 4.3 (3.6) and 3.9 (2.7) supernumerary embryos have been cryopreserved, respectively.

In the corifollitropin alfa group $29.2 \%(221 / 756)$, and in the $\mathrm{rFSH}$ group $30.3 \%$ (227/750), received only i.m. luteal phase progesterone support, whereas respectively $52.0 \%(393 / 756)$ and $54.9 \%(412 / 750)$ received only intravaginal progesterone support. Other subjects received a combination of routes.

Pregnancy rates (confirmed by positive hCG test) of 48.1 and $46.9 \%$ were achieved in the corifollitropin alfa and $\mathrm{rFSH}$ groups, respectively, followed by a comparable number of early pregnancy losses during the first 10 weeks after embryo transfer. Accordingly, similar ongoing pregnancy rates were observed in both groups (Table IV). There was no relevant difference in the ongoing pregnancy rates between subjects who received the hCG injection on the same day that three follicles $\geq 17 \mathrm{~mm}$ were observed ( 40.0 and $37.8 \%$ for corifollitropin alfa and $\mathrm{rFSH}$, respectively) versus subjects who received hCG one day later (38.9 and $41.8 \%$ for corifollitropin alfa and $\mathrm{rFSH}$, respectively).

The robustness of this primary efficacy outcome was explored in specific subsets of patients grouped according to the ART procedure-related factors, i.e. undergoing IVF or ICSI, having single or double embryo transfer and having embryo transfer on day 3 or day 5 (Fig. 3). The ongoing pregnancy rates in each subset were similar for corifollitropin alfa and $\mathrm{rFSH}$ and there was no significant interaction with the type of treatment factor (IVF or ICSI), nor for
Table IV Clinical efficacy outcomes per started cycle (intent-to-treat population)

\begin{tabular}{|c|c|c|c|}
\hline & $\begin{array}{l}\text { Corifollitropin } \\
\text { alfa }(n=756)\end{array}$ & $\begin{array}{l}\text { rFSH } \\
(n=750)\end{array}$ & $P$-value ${ }^{\text {a) }}$ \\
\hline Positive hCG test ${ }^{\text {b) }}, n(\%)$ & $364(48.1)$ & $352(46.9)$ & 0.64 \\
\hline Clinical pregnancy ${ }^{c}, n(\%)$ & $322(42.6)$ & $308(4 I . I)$ & 0.57 \\
\hline Vital pregnancy ${ }^{\mathrm{d})}, n(\%)$ & $302(39.9)$ & $293(39.1)$ & 0.75 \\
\hline Ongoing pregnancy ${ }^{\mathrm{e})}, n(\%)$ & $294(38.9)$ & $286(38.1)$ & 0.71 \\
\hline Multiple pregnancy ${ }^{f}, n(\%)$ & $83(28.2)$ & $66(23.1)$ & 0.18 \\
\hline Early miscarriage $^{\mathrm{g})}, n(\%)$ & $27(8.4)$ & $21(6.8)$ & 0.55 \\
\hline
\end{tabular}

a) $P$-values are based on Fisher's exact test, except for ongoing pregnancy where the $P$-value is based on the likelihood ratio test corresponding to the generalized linear model with covariates treatment group, age class ( $<32$ years, $\geq 32$ years) and region (Europe, North America).

b) Positive hCG test at least 14 days after embryo transfer or USS with at least one gestational sac.

${ }^{c}$ Clinical pregnancy: gestational sac on USS.

d) Vital pregnancy: gestational sac + fetal heartbeat.

e) Ongoing pregnancy: vital fetus at least 10 weeks after embryo transfer or live birth.

f) Per ongoing pregnancy.

${ }^{\text {g) }}$ Per clinical pregnancy.

the number of embryos transferred (one or two). A borderline significant interaction with the type of treatment was only observed for the factor day of transfer $(P=0.045)$ as a result of higher pregnancy rates for day 3 transfers with corifollitropin alfa whereas, for day 5 transfers, the $\mathrm{rFSH}$ group showed marginally higher pregnancy rates (Fig. 3). The subset analyses together confirm the robustness of the observed difference in pregnancy rates, independent of ART procedure-related parameters.

Multiple pregnancy rates per ongoing pregnancy were $28.2 \%$ and $23.1 \%$ in the corifollitropin alfa group and the $\mathrm{rFSH}$ group, respectively (Table IV). Although this difference is not significant, it is in line with the slightly higher implantation rate defined as 100 times the maximum number of gestational sacs divided by the number of embryos transferred per subject for corifollitropin alfa [observed means (SD) were $36.2 \%$ (41.6\%) versus $32.2 \%$ (40.1\%)].

\section{Safety}

In total, 16 subjects $(2.1 \%)$ in the corifollitropin alfa group discontinued due to a (serious) adverse event [SAE, two (0.3\%) before and I 4 (I.9\%) following oocyte retrieval] as compared with three subjects $(0.4 \%)$ in the rFSH group [two $(0.3 \%)$ before and one $(0.1 \%)$ after oocyte retrieval]. A total of 53 patients in the corifollitropin alfatreated group $(7.0 \%)$ and 47 patients in the rFSH-treated group (6.3\%) developed OHSS in this trial. The incidences of (moderate/ severe) OHSS were $4.1 \%$ [3I/755, 95\% Cl: $(2.6 ; 5.6)]$ and $2.7 \%$ [20/750, 95\% Cl: $(1.5 ; 3.9)]$ for the corifollitropin alfa and $r F S H$ group, respectively, which was not significant (Fisher exact $P=0.15$ ).

An equal number of 37 SAEs was reported for the corifollitropin alfa and the $\mathrm{rFSH}$-treated groups. Most frequently reported SAEs were OHSS [14 patients (1.9\%) treated with corifollitropin alfa and 9 patients ( $1.2 \%$ ] treated with rFSH) and (ruptured) ectopic pregnancy [7 patients $(0.9 \%)$ and 9 patients ( $1.2 \%)$, respectively] while, respectively, the most frequently reported AEs were procedural pain (22.3 


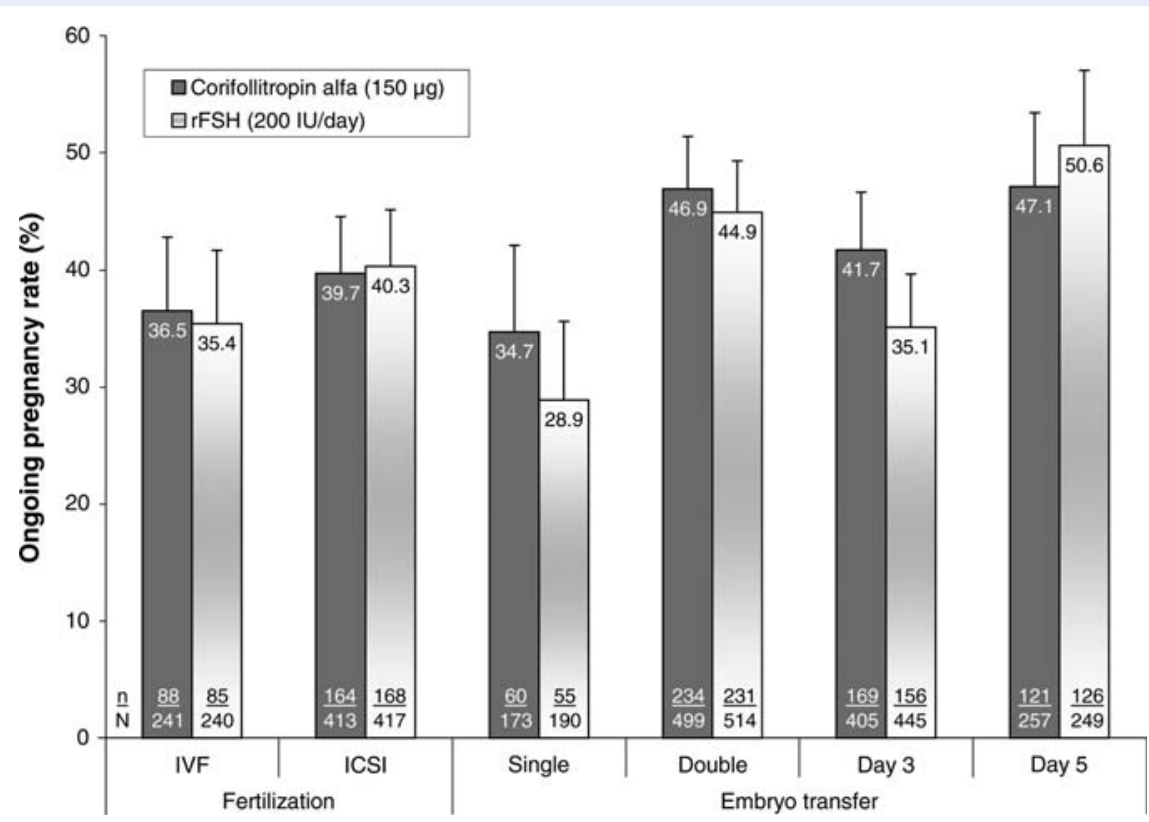

Figure 3 Ongoing pregnancy rates stratified for fertilization procedure, number of embryos transferred and day of transfer. Subset with double embryo transfer includes three patients with three embryos transferred.

and $20.1 \%$ ), pregnancy-related events including (missed) abortion (I3.8 and II.2\%), pelvic pain ( 12.1 and $12.3 \%)$, pelvic discomfort (II.5 and $11.6 \%)$ and headache (10.5 and $15.2 \%)$. There were no drug-related immune responses or moderate or severe local tolerance reactions observed in this trial.

\section{Discussion}

The current ENGAGE trial was a double-blind, double-dummy trial initiated to investigate whether the new corifollitropin alfa regimen provides similar success rates compared with the current care. The double-dummy approach guaranteed the blinding of medication during the trial and prevented any bias in terms of treatment decisions. Owing to the fact that the trial was powered to enable comparison of the ongoing pregnancy rates as the primary study end-point, this is the largest double-blind efficacy trial in IVF performed to date. The outcome of the trial provides compelling evidence of equal efficacy in terms of ongoing pregnancy rates, because the point estimates i.e. 38.9 and $38.1 \%$ for the ongoing pregnancy rates were very close for the two treatment groups and the $95 \%$ lower limit of the difference was only $-3.9 \%$. Subset analyses of subjects undergoing IVF or ICSI and of subjects who had single or double embryo transfer reveal consistently high ongoing pregnancy rates, similar between the treatment groups, which confirm the robustness of the primary end-point. Even though an equal number and quality of embryos were replaced in both treatment groups, the multiple pregnancy rate in the corifollitropin alfa group tended to be higher $(+4.4 \%$ absolute risk increase $)$ than in the reference group, which may be related to the slightly higher implantation rate. This increase, although not significant, cannot be ruled out and may prove to be clinically relevant, given the low power of the study to detect such a difference (c.22\%). In this context, it should be emphasized that both treatment arms used a 'protocolized' fixed dose treatment regimen applying $\mathrm{GnRH}$ antagonist (ganirelix) co-treatment, which confirms the successful outcome of a patientfriendly short $\mathrm{GnRH}$ antagonist protocol in corifollitropin alfa, as well as $\mathrm{rFSH}$, treatment cycles. In addition, one-third of the patients treated with a single injection of corifollitropin alfa reached the criteria for hCG injection prior to or on stimulation day 8 , omitting the need for any additional FSH injections. The ongoing pregnancy rate of this subset of good responder patients was $44.0 \%$, thus $5.9 \%$ higher than the overall group of subjects treated with corifollitropin alfa.

A hybrid molecule composed of human FSH and the CTP of hCG was first described by Boime and colleagues (Fares et al., 1992). In subsequent clinical trials the new recombinant fertility hormone exhibited a slower absorption (and subsequent rise to peak levels) and an approximately 2-fold longer $t_{1 / 2}$ compared with rFSH (Bouloux et al., 200I; Duijkers et al., 2002). After corifollitropin alfa injection, peak levels of FSH activity are reached within 2 days whereas steady state levels with daily FSH are reached only after 4-5 days (Mannaerts et al., 1996). These pharmacokinetic properties of corifollitropin alfa create an opportunity to further simplify ovarian stimulation protocols for IVF by omitting the need for daily gonadotrophin injections during the first week of stimulation (Fauser et al., 2009). In the current trial, the difference in exposure during the first days of stimulation may have resulted in a slightly higher ovarian response in the corifollitropin alfa treated patients, as previous trials with $\mathrm{rFSH}$ have shown that the number of follicles recruited increases with the starting dose of rFSH given (Wikland et al., 200I; Out et al., 2004). In view of the equal pregnancy rates, the current trial does not suggest that the higher exposure to FSH immunoactivity during the first days of stimulation interferes with the endometrial receptivity. 
In this trial hormonal pretreatment with oral contraceptives or supplementation with LH or hCG during the treatment phase was not allowed per protocol. The efficacy results in this large, global, multi-center trial suggest that the amount of endogenous $\mathrm{LH}$ during treatment with either corifollitropin alfa or daily rFSH in a standard $\mathrm{GnRH}$ antagonist protocol is sufficient to support high success rates in terms of ongoing pregnancies (Cedrin et al., 2004).

Because of concerns that the relatively high exposure to corifollitropin alfa during the first days of stimulation might initiate an early rise of LH (Devroey et al., 2004), all patients in this trial started treatment with ganirelix on stimulation day 5. This fixed start of $\mathrm{GnRH}$ antagonist co-treatment was considered advantageous from an efficacy perspective as this may result in higher pregnancy rates, as well as from a methodological point of view as this reduces variability in the applied treatment regimens (Kolibianakis et al., 2004; Al-Inany et al., 2005; Arce et al., 2005).

The corifollitropin alfa regimen used in this trial comprised a single dose of corifollitropin alfa followed by daily doses of $r F S H$ (as needed up to a daily maximum of $200 \mathrm{IU}$ ). This corifollitropin regimen was compared with a fixed dose of $200 \mathrm{IU} /$ day $\mathrm{rFSH}$ for the first 7 days, but with the option to decrease the daily FSH dose in cases when hyper-response was observed. Thus there was a difference in patient management options, with no option to adjust the dosing during the first week of stimulation in the corifollitropin arm. After corifollitropin alfa injection, serum FSH activity declines from stimulation day $3\left(C_{\max }\right)$ onwards, but further reduction of exposure during the first week of stimulation cannot be attained.

The validity of the claimed efficacy and safety data of this trial are limited to the study population only. Patients with known risk factors for a hyper-response, such as patients with a history of OHSS, with PCOS or with a high AFC $(>20)$ were excluded from the current trial. In addition, patients with a history of low ovarian response in a previous IVF cycle were excluded. However, it should be noted that $74 \%$ of the patients included in this trial underwent their first IVF cycle for which the ovarian response is less predictable and will still include low- and high responder patients.

In this large cohort of (potential) normal responder patients, corifollitropin alfa has been shown to be well tolerated and non-immunogenic. In line with the higher ovarian response, the observed incidence of OHSS was higher in the corifollitropin alfa group, but the difference was not significant. Our study was not sufficiently powered to detect an underlying difference in the incidence of OHSS, and therefore its actual presence cannot be excluded. In line with the recruitment of slightly more follicles by corifollitropin alfa, dose reductions during stimulation were more frequently made in the corifollitropin alfa group than in the reference group. Clearly, a minority of subjects required a dose reduction on stimulation day 6 or 7 , which may have affected the ovarian response in the $\mathrm{rFSH}$ group rather than in the corifollitropin alfa group in which only the placebo was reduced.

Appropriate clinical monitoring and lowering or withholding the daily rFSH dose to complete the cycle, as well as lowering or withholding the dose of hCG to trigger final oocyte maturation, or cryopreservation of all embryos obtained are options which can be considered as part of patient management to minimize the risk of OHSS (Delvigne and Rozenberg, 2002).

Owing to the double-blind design of the trial, requiring all patients to be treated with an equal number of injections, no comparative data could be collected on perceived patient convenience or preference for the corifollitropin alfa regimen. Since a single injection of corifollitropin alfa replaces the first seven daily injections of $\mathrm{rFSH}$ during ovarian stimulation, the intuitive advantages of such a simpler treatment regimen are obvious, but need to be confirmed in clinical practice. In addition to patient preference, future controlled trials may examine the efficacy of corifollitropin alfa in (potential) poor responding patients as well as the safety in (potential) high responders.

In conclusion, in this study we tested the efficacy (in terms of ongoing pregnancy rates) of substituting the first 7 daily doses of rFSH with a single injection of corifollitropin alfa in women undergoing ovarian stimulation for IVF/ICSI using $\mathrm{rFSH}$ and $\mathrm{GnRH}$ antagonists. Our data demonstrate that a single corifollitropin alfa injection results in an ongoing pregnancy rate which is equal to that of a daily rFSH regimen. Combined with appropriate patient selection and state-of-the-art clinical management during the stimulation phase of the treatment cycle, corifollitropin alfa potentially offers an attractive new treatment option for patients undergoing ovarian stimulation during ART.

\section{Acknowledgements}

The authors thank the participating patients, ENGAGE investigators and clinical staff. Medical writing support was provided by Patrick Langford at Prime Medica Ltd during the preparation of this paper, supported by Schering-Plough. Responsibility for opinions, conclusions and interpretation of data lies with the authors.

Conflict of interest: P.D. has received fees and grants from the following companies: organon, Schering-Plough, Ferring, Bessins, Serono and IBSA. R.B. has received fees and grants from the following companies: Organon, Schering-Plough, Merck Serono and Ferring. B.C.J.M.F. has received fees and grants from the following companies: Organon, Schering-Plough, Serono, Merck Serono, Ferring, Wyeth, Schering, Ardana, Andromed, Pantarhei Bioscience and Preglem. N.P.K., B.M.J.L. M. and P.C.I.B. are employees of Schering-Plough. These data were presented in part at the Annual ASRM meeting 2008 (San Francisco (CA), USA) and ESHRE 2009 (Amsterdam, The Netherlands).

\section{Funding}

Financial support was provided by the Schering-Plough Research Institute, Schering-Plough, Oss, The Netherlands.

\section{References}

Al-Inany H, Aboulghar MA, Mansour RT, Serour Gl. Optimizing GnRH antagonist administration: meta-analysis of fixed versus flexible protocol. Reprod Biomed Online 2005; 10:567-570.

Arce JC, Nyboe Andersen A, Collins J. Resolving methodological and clinical issues in the design of efficacy trials in assisted reproductive technologies: a mini-review. Hum Reprod 2005;20: I757- 177I.

Boivin J, Takefman JE. Impact of the in-vitro fertilization process on emotional, physical and relational variables. Hum Reprod 1996; I I:903-907.

Bouloux PM, Handelsman DJ, Jockenhövel F, Nieschlag E, Rabinovici J, Frasa WL, de Bie JJ, Voortman G, Itskovitz-Eldor J, FSH-CTP study 
group. First human exposure to FSH-CTP in hypogonadotrophic hypogonadal males. Hum Reprod 200 I; 16:1592-1597.

Cédrin-Durnerin I, Grange-Dujardin D, Laffy A, Parneix I, Massin N, Galey J, Théron L, Wolf JP, Conord C, Clément $\mathrm{P}$ et al. Recombinant human $\mathrm{LH}$ supplementation during $\mathrm{GnRH}$ antagonist administration in IVF/ICSI cycles: a prospective randomized study. Hum Reprod 2004; 19:1979-1984.

Cousineau TM, Domar AD. Psychological impact of infertility. Best Pract Res Clin Obstet Gynaecol 2007;21:293-308.

De Greef R, Struijs M, de Haan A, Marintcheva-Petrova M, Mannaerts BMJL. Dose selection of Org 36286 (corifollitropin alfa) using pharmacokinetic and dynamic modeling and simulation. Hum Reprod 2007;22:139 (Abstract O-099).

Delvigne A, Rozenberg S. Epidemiology and prevention of ovarian hyperstimulation syndrome (OHSS): a review. Hum Reprod Update 2002;8:559-577.

Devroey P, Fauser BC, Platteau P, Beckers NG, Dhont M, Mannaerts BM. Induction of multiple follicular development by a single dose of long-acting recombinant follicle-stimulating hormone (FSH-CTP, corifollitropin alfa) for controlled ovarian stimulation before in vitro fertilisation. J Clin Endocrinol Metab 2004;89:2062-2070.

Domar AD, Zuttermeister PC, Friedman R. Psychological impact of infertility: a comparison with patients with other medical conditions. J Psychosom Obstet Gynaecol 1993; 14:45-52.

Duijkers JJM, Klipping C, Boerrigter PJ, Machielsen CSM, de Bie JJ, Voortman G. Single dose pharmacokinetics and effects on follicular growth and serum hormones of a long-acting recombinant FSH preparation (FSH-CTP) in healthy pituitary-suppressed females. Hum Reprod 2002; 17:1987-1993.

Fares FA, Suganuma N, Nishimori K, LaPolt PS, Hsueh AJ, Boime I. Design of a long-acting follitropin agonist by fusing the $\mathrm{C}$-terminal sequence of the chorionic gonadotropin beta subunit to the follitropin beta subunit. Proc Natl Acad Sci USA 1992;89:4304-4308.

Fauser BCJM, Mannaerts BMJL, Devroey P, Leader A, Boime I, Baird DT. Advances in recombinant DNA technology: corifollitropin alfa, a hybrid molecule with sustained follicle-stimulating activity and reduced injection frequency. Hum Reprod Update 2009 (E Pub Ahead of Print).

Gleicher N, Weghofer A, Barad D. A formal comparison of the practice of assisted reproductive technologies between Europe and the USA. Hum Reprod 2006;21:1945-1950.

Gleicher N, Weghofer A, Barad D. Update on the comparison of assisted reproduction outcomes between Europe and the USA: the 2002 data. Fertil Steril 2007;87:1301-1305.

Hammarberg K, Astbury J, Baker HWG. Women's experience of IVF: a follow-up study. Hum Reprod 200 I; 16:374-383.

Heijnen EM, Macklon NS, Fauser BCJM. What is the most relevant standard of success in assisted reproduction? The next step to improving outcomes of IVF: consider the whole treatment. Hum Reprod 2004; 19:1936-1938.

Kolibianakis EM, Zikopoulos K, Schiettecatte J, Smitz J, Tournaye H, Camus M, Van Steirteghem AC, Devroey P. Profound LH suppression after $\mathrm{GnRH}$ antagonist administration is associated with a significantly higher ongoing pregnancy rate in IVF. Hum Reprod 2004; 1 9:2490-2496.

Land JA, Courtar DA, Evers JLH. Patient dropout in an assisted reproductive technology program: implications for pregnancy rates. Fertil Steril 1997;68:278-28I.

LaPolt PS, Nishimori K, Fares FA, Perlas E, Boime I, Hsueh AJ. Enhanced stimulation of follicle maturation and ovulatory potential by long acting follicle-stimulating hormone agonists with extended carboxyl-terminal peptides. Endocrinology 1992; 13 I:25|4-2520.

Mannaerts BMJL, Shoham Z, Schoot D, Bouchard P, Harlin J, Fauser BCJM, Jacobs H, Rombout F, Coelingh Bennik H. Single-dose pharmacokinetics and pharmacodynamics of recombinant human follicle-stimulating hormone (Org 32849) in gonadotropin-deficient volunteers. Fertil Steril 1993;59:108-||14.

Mannaerts BMJL, Rombout F, Out HJ, Coelingh Bennink HJT. Clinical profiling of recombinant follicle stimulating hormone (rFSH; Puregon): relationship between serum FSH and efficacy. Hum Reprod Update 1996;2:153-161.

Olivennes F. Patient-friendly ovarian stimulation. Reprod Biomed Online 2003; 7:30-34.

Olivius C, Friden B, Lundin K, Bergh C. Cumulative probability of live birth after three in vitro fertilization/intracytoplasmic sperm injection cycles. Fertil Steril 2002;77:505-5I0.

Olivius C, Friden B, Borg G, Bergh C. Why do couples discontinue in vitro fertilisation treatment? A cohort study. Fertil Steril 2004; 8I:258-26I.

Out HJ, Rutherford A, Fleming R, Tay CC, Trew G, Ledger W, Cahill D. A randomized, double-blind, multicentre clinical trial comparing starting doses of I50-200 IU of recombinant FSH in women treated with the $\mathrm{GnRH}$ antagonist ganirelix for assisted reproduction. Hum Reprod 2004; 19:90-95.

Papanikolaou EG, Pozzobon C, Kolibianakis EM, Camus M, Tournaye H, Fatemi HM, Van Steirteghem AC, Devroey P. Incidence and prediction of ovarian hyperstimulation syndrome in women undergoing gonadotropin-releasing hormone antagonist in vitro fertilization cycles. Fertil Steril 2006;85: I |2-120.

Pennings G, Ombelet W. Coming soon to your clinic: patient-friendly ART. Hum Reprod 2007;22:2075-2079.

Rajkhowa M, Mcconnell A, Thomas GE. Reasons for discontinuation of IVF treatment: a questionnaire study. Hum Reprod 2006;2 I: 358-363.

Schröder AK, Katalinic A, Diedrich K, Ludwig M. Cumulative pregnancy rates and drop-out rates in a German IVF programme: 4I02 cycles in 2130 patients. Reprod Biomed Online 2004;8:600-606.

Tarlatzis BC, Fauser BCJM, Kolibianakis EM, Diedrich K, Devroey P, on behalf of the Brussels GnRH Antagonist Consensus Workshop Group. GnRH antagonists in ovarian stimulation for IVF. Hum Reprod Update 2006; I 2:333-340.

The Corifollitropin Alfa Dose-Finding Study Group. A randomized dose-response trial of a single injection of corifollitropin alfa to sustain multifollicular growth during controlled ovarian stimulation. Hum Reprod 2008;23:2484-2492.

Van der Gaast M, Eijkemans MJC, Van der Net JB, de Boer EJ, Burger CW, Van Leeuwen FE, Fauser BCJM, Macklon NS. Optimum number of oocytes for a successful first IVF treatment cycle. Reprod Biomed Online 2006; 13:476-480.

Verberg MF, Eijkemans MJ, Heijnen EM, Broekmans FJ, de Klerk C, Fauser BCJM, Macklon NS. Why do couples drop out from IVF treatment? A prospective cohort study. Hum Reprod 2008; 23:2050-2055.

Verwoerd GR, Mathews T, Brinsden PR. Optimal follicle and oocyte numbers for cryopreservation of all embryos in IVF cycles at risk of OHSS. Reprod Biomed Online 2008; 17:312-317.

Wikland M, Bergh C, Borg K, Hillensjo T, Howles CM, Knutsson A, Nilsson L, Wood M. A prospective, randomized comparison of two starting doses of recombinant FSH in combination with cetrorelix in women undergoing ovarian stimulation for IVF/ICSI. Hum Reprod 200I;16:1676-168|.

WHO. Agents stimulating gonadal function in the human. World Health Organ Tech Rep Ser 1973;5 14:1-30.

Submitted on February 17, 2009; resubmitted on July 14, 2009; accepted on July 20, 2009 